\title{
Identification of N-Glycosylation Sites with Sequence and Structural Features Employing Random Forests
}

\author{
Shreyas Karnik ${ }^{1,3}$, Joydeep Mitra ${ }^{1}$, Arunima Singh ${ }^{1}$, B.D. Kulkarni ${ }^{1}$, \\ V. Sundarajan ${ }^{2}$, and V.K. Jayaraman ${ }^{2, \star}$ \\ ${ }^{1}$ Chemical Engineering and Process Development Division, National Chemical \\ Laboratory, Pune, India - 411008 \\ 2 Center for Development of Advanced Computing, Pune University Campus, Pune, \\ India - 411007 \\ jayaramanv@cdac.in \\ 3 School of Informatics, Indiana University, Indianapolis, IN, USA, 46202
}

\begin{abstract}
N-Glycosylation plays a very important role in various processes like quality control of proteins produced in ER, transport of proteins and in disease control.The experimental elucidation of $\mathrm{N}-$ Glycosylation sites is expensive and laborious process. In this work we build models for identification of potential N-Glycosylation sites in proteins based on sequence and structural features. The best model has cross validation accuracy rate of $72.81 \%$.
\end{abstract}

\section{Introduction}

Carbohydrates that are attached to proteins play various important roles in biological systems. One of the major functions of protein linked glycans is to provide additional epitopes for the sake of recognition by protein receptors. 12 This type of recognition events are involved in a myriad of processes such as initiation of inflammation, protein trafficking and defense mechanisms [3.N-glycosylation refers to the addition of oligomeric glycans to asparagine (ASN or N) residues on proteins. The sequence motif $\operatorname{Asn}(\mathrm{N})-\mathrm{Xaa}-\operatorname{Ser}(\mathrm{S}) / \mathrm{Thr}(\mathrm{T})$ has been defined are prerequisite for N-glycosylation. In the motif N-X-S/T, X position can be occupied by any amino acid except for proline 4. In some cases of N-Glycosylation the motif N-X-C (Cystine) is also found but vary rarely. The presence of $\mathrm{N}-\mathrm{X}$ $\mathrm{S} / \mathrm{T}$ motif is a necessary but not sufficient criterion for N-Glycosylation.

Experimental determination of N-glycosylation in proteins is a laborious and expensive process [5]. Although methods like X-ray crystallography provide direct and unambiguous evidence for the glycan occupied sites, evidence from X-ray crystallography for the unoccupancy of a site is more ambiguous because the absence of the glycan is only one reason for the absence of resolved electron density. Thus, there is need for computational approaches in determining the

^ Corresponding Author

S. Chaudhury et al. (Eds.): PReMI 2009, LNCS 5909, pp. 146-151 2009.

(C) Springer-Verlag Berlin Heidelberg 2009 
glycosylation of proteins from the available sequence and structural information. Machine learning methods are explored to provide reliable and cost effective predictive model based on the known data of the proteins several approaches that handle this problem.Gupta et al. [6] have used sequence features for the prediction of potential N-Glycosylation sites in humans using neural networks. In a recent study Caragea et. al[7] have used ensemble of Support Vector Machines (SVM) classifiers using string kernels to identify potential glycosylated sites. In their comprehensive study on protein environment of N-glycosylation sites Petrescu et al. and Ben-Dor et al. 5 [5] have indicated that the sequence and structural properties play an important role in deciding the occupancy of the sequon. They came up with the following observations in the analysis of the N-Glycosylation sites :

1. Glycosylation sites occur on hydrophobic regions of the protein surface.

2. Marked preference for hydrophobic residues at both the sides on the glycosylated sites.

3. There is a clear preference for nonpolar amino acids in positions +2 to +4 and in particular for aromatic residues in position +2 and +1 , small nonpolar amino acids in position +1 , and bulky hydrophobic amino acids in positions +3 to +5 .

4. There is a notable reduction in the probability of finding acidic residues immediately before the glycosylation site.

5. When secondary structure around these sites was analyzed they found a bias in favor of turns and bends.

Apart from the above mentioned factors, accessibility and conformational factors play an important role in the process of glycosylation. It is clear that identification of structural and sequence features that correlate with N-Glycosylation is important. In our study we have employed Random Forest(RF) [14] classifier for selection of the most important features and prediction of N-Glycosylation sites in proteins.

\section{Materials and Methods}

Collection of Data: "ASN GLYCOSYLATION" was used as keyword for advanced search at the RCSB PDB[9] server. 1671 hits were found which were filtered at $90 \%$ sequence similarity and the selected 465 structures were downloaded. Occupied glycosylation sites (NXS/T) in each structure were determined by the MODRES field in the PDB file while the unoccupied sites were found by scanning the structure for NXS/T sites other than the ones mentioned in the MODRES field. For the negative dataset we used PDB select 25 data and all the $\mathrm{N}-\mathrm{X}-\mathrm{S} / \mathrm{T}$ sites, which are not glycosylated, were taken. From the positive and the negative sequences a window of fixed length $(11,19$ and 35$)$ with(N-X-S/T) at the center flanked by equal number of amino acids on both sides were extracted. After the collection of the subsequences containing the glycosylated sites the task at hand was to quantify the innate patterns in the sequences into features 
that can be used as knowledge by the machine learning algorithm to identify glycosylation sites. We considered to use the amino acid frequencies to begin with but literature sources [5] indicated that the process of glycosylation has a interplay at the compositional and the structural levels so we incorporated this information in terms of sequence and structure based features described below. We have used Random Forest as the classification algorithm which also has the ability to select the most informative features from a large number of features, because of this capability of Random Forests we used a variety of features that can help us gain insights into the signals in the subsequences that are responsible for the sequon to get glycosylated. We have used features that represent sequence and structure related information of glycosylated subsequences.

The sequence and structural features comprises of the following features:

- Sequence Dependant Features

- Amino acid and Dipeptide frequencies

- Features based on physiochemical properties of amino acids by the PROFEAT Server 10 ]

- Structural Features

- Secondary Structure assignment from DSSP 11

- Solvent Accessibility

- Contact Number

- Contact Order

- Backbone Torsional Angles

The PROFEAT Server 10 calculates different autocorrelation descriptors these are defined on the basis of of the distribution of the different amino acid properties along the sequence. These autocorrelation descriptors for a particular property can provide insights about the periodicity of amino acid physiochemical properties. Amino acid properties employed for the computation of autocorrelation descriptors were taken from AAIndex [12. A detailed list of the amino acid properties used for the calculation of autocorrelation descriptors is given in Table I.We have used Random Forest 13 to build a model that will aid in the identification of the N-Glycosylated sites from the two set of the features described above. Random Forest is an algorithm for classification and regression developed by Leo Breiman[14 that uses an ensemble of trees for classification and regression. Each of the classification trees is built using a bootstrap sample of the data, and at each split the candidate set of variables is a random subset of the variables (mtry). Thus, Random Forest uses both bagging (bootstrap aggregation), a successful approach for combining unstable learners, and random variable selection for tree building. Each tree is unpruned (grown fully). The trees grown then vote on the class for a given input, the class that gets the maximum number of votes is then assigned to the input. Random forest has excellent performance in classification tasks. It has been successfully employed to various problems from the life sciences domain 15,16 17/18] it has several characteristics that make it ideal for these data sets. We used the Random Forest implementation in R[19] by Andy Liaw and Matthew Wiener 20] based on original code in FORTRAN by Breiman. The methodology applied for the selection of the best features is as follows: 
1. Start with the full set of features.

2. Tune the mtry parameter to find the optimal value (usually in the vicinity of the square root of the total number of features).

3. Once an optimal value of mtry has been found build Random Forest model and get the variable importance measure for each feature. Also do 10-fold cross-validation in this step.

4. Select half the number of top scoring features and build Random Forest model again. Monitor Mathew's Correlation Coefficient (MCC) on the training dataset each time.

5. Repeat steps 2-4 till the highest MCC is obtained.

\section{Results and Discussion}

The study attempts to build a model for identification of N-Glycosylation sites using Random Forests Classifier. We used sequence and structure dependent features for building models that could identify the N-Glycosylation sites with good accuracy. We used the subsequence lengths of 11,19 and 35 for feature calculation. Models for the classification of N-Glycosylation sites using different subsequence lengths generated using the methodology described above. In all the models the model with subsequence length 35 and 28 features (using feature selection) performed best. This model has accuracy $72.81 \%$ and MCC 0.4426 ; summary of this model is given in Table 1.

Table 1. Summary of the Best Model

\begin{tabular}{l|l|l|l|l|l|l|l|l}
\hline Model Description & Accuracy & $\mathrm{TP}^{a}$ & $\mathrm{TN}$ & $\mathrm{FP}$ & $\mathrm{FN}$ & Sensitivity & Specificity & $\mathrm{MCC}$ \\
\hline Model $1^{b}$ & 72.81 & 591 & 590 & 212 & 211 & 0.716 & 0.735 & 0.442 \\
\hline
\end{tabular}

${ }^{a}$ TP:-True Positives, TN:- True Negatives,FP:- False Positives, FN:- False Negatives.

${ }^{b}$ Best Model:- Comprises of top 28 structural and sequence features (subsequence length 35).

The window length 35 is giving good performance signifies that there are long term interactions that play a very important role in the Glycosylation process. It turns out that the in the sequence and the structural features contact number, contact order, solvent accessibility, composition descriptor of hydrophobic amino acids and Geary autocorrelation descriptors [10] around the glycosylated site play an important role in determining the occupancy of the sequon the list of top 10 features is given in Table 2.

The top scoring features of this model agree well with a comprehensive study that does the statistical analysis of the N-glycosylated sites [58]. There have been different significant approaches for the identification of N-Linked Glycosylated sites by Gupta et al. [6] who reported 76\% accuracy for the identification of N-Glycosylation sites in humans and Caragea et al. [7] who employed ensembles of Support Vector Machines for the prediction of Glycosylation sites (N-linked,O-linked and C-mannosylation) in this work the accuracy for N-linked 
Table 2. Top 10 features from the model incorporating Sequence and Structural Features

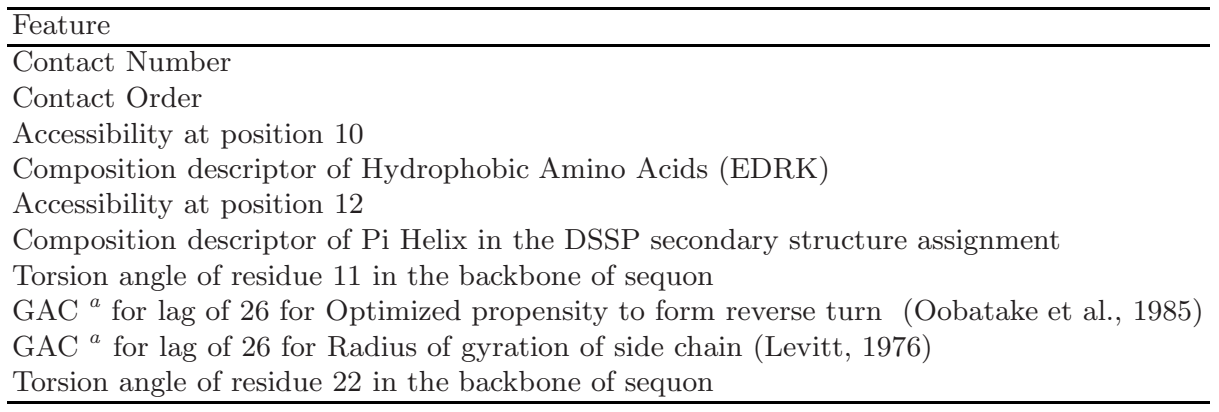

${ }^{a}$ GAC:- Geary Autocorrelation Descriptor.

glycosylation with Single SVM is 94\%, but contains less number of experimentally verified N-Glycosylation sites thus, a rational comparison of our approach with these methodologies is not possible owing to the difference in the datasets and methodologies.

\section{Conclusion}

The aim of this study is to provide a reliable model to predict the N-linked glycosylation of sequons employing Random Forest classifier. Apart from classification, Random Forest also provides heuristic information in terms of a ranking score. This facilitates us to select the top ranking features to obtain optimal classification performance. The latter provides valuable domain knowledge regarding the N-Glycosylated sites. Finally, the model with sequence and structural features has a subsequence length of 35 and a MCC of 0.4426 and accuracy 72.81 with sensitivity as 0.7165072 and specificity as 0.7356771 . This type of study provides a high throughput screening mechanism in experiments where the role of N-Glycosylation is being investigated in some functional aspect, in this case this model will provide reliable first hand results even before the N-Glycosylation is experimentally verified.

Acknowledgements. VKJ gratefully acknowledes financial assistance from Department of Science and Technology New Delhi, INDIA. VKJ also acknowledges fruitful suggestions by Dr. Petety V. Balaji, IIT, Mumbai, INDIA.

\section{References}

1. Drickamer, K., Taylor, M.E.: Biology of animal lectins. Annual Review of Cell Biology 9(1), 237-264 (1993) PMID: 8280461

2. Lis, H., Sharon, N.: Lectins: Carbohydrate-specific proteins that mediate cellular recognition. Chemical Reviews 98(2), 637-674 (1998) 
3. Crocker, P.R.: Siglecs: sialic-acid-binding immunoglobulin-like lectins in cell-cell interactions and signalling. Curr. Opin. Struct. Biol. 12(5), 609-615 (2002)

4. Gavel, Y., Heijne, G.v.: Sequence differences between glycosylated and non- glycosylated Asn-X-Thr/Ser acceptor sites: implications for protein engineering. Protein Eng. 3(5), 433-442 (1990)

5. Petrescu, A.J., Milac, A.L., Petrescu, S.M., Dwek, R.A., Wormald, M.R.: Statistical analysis of the protein environment of $\mathrm{N}$-glycosylation sites: implications for occupancy, structure, and folding. Glycobiology 14(2), 103-114 (2004)

6. Gupta, R., Jung, E., Brunak, S.: Netnglyc 1.0 server (Unpublished)

7. Caragea, C., Sinapov, J., Silvescu, A., Dobbs, D., Honavar, V.: Glycosylation site prediction using ensembles of support vector machine classifiers. BMC Bioinformatics 8, 438-438 (2007)

8. Ben-Dor, S., Esterman, N., Rubin, E., Sharon, N.: Biases and complex patterns in the residues flanking protein N-glycosylation sites. Glycobiology 14(2), 95-101 (2004)

9. Sussman, J.L., Lin, D., Jiang, J., Manning, N.O., Prilusky, J., Ritter, O., Abola, E.E.: Protein data bank (pdb): database of three-dimensional structural information of biological macromolecules. Acta Crystallogr. D. Biol. Crystallogr. 54, 10781084 (1998)

10. Li, Z.R., Lin, H.H., Han, L.Y., Jiang, L., Chen, X., Chen, Y.Z.: PROFEAT: a web server for computing structural and physicochemical features of proteins and peptides from amino acid sequence. Nucl. Acids Res. 34, W32-W37 (2006)

11. Kabsch, W., Sander, C.: Dictionary of protein secondary structure: pattern recognition of hydrogen-bonded and geometrical features. Biopolymers 22(12), 2577-2637 (1983)

12. Kawashima, S., Pokarowski, P., Pokarowska, M., Kolinski, A., Katayama, T., Kanehisa, M.: AAindex: amino acid index database, progress report. Nucl. Acids Res. 36, D202-D205 (2008)

13. Breiman, L.: Random forests. Machine Learning, 5-32 (2001)

14. Breiman, L.: Random forests. Machine Learning 45(1), 5-32 (2001)

15. Bureau, A., Dupuis, J., Falls, K., Lunetta, K.L., Hayward, B., Keith, T.P., Van Eerdewegh, P.: Identifying SNPs predictive of phenotype using random forests. Genetic Epidemiology 28(2), 171-182 (2005)

16. Diaz-Uriarte, R., Alvarez de Andres, S.: Gene selection and classification of microarray data using random forest. BMC Bioinformatics 7(1), 3 (2006)

17. Hamby, S., Hirst, J.: Prediction of glycosylation sites using random forests. BMC Bioinformatics 9, 500 (2008)

18. Pang, H., Lin, A., Holford, M., Enerson, B.E., Lu, B., Lawton, M.P., Floyd, E., Zhao, H.: Pathway analysis using random forests classification and regression. Bioinformatics (2006)

19. R Development Core Team: R: A Language and Environment for Statistical Computing. In: R. Foundation for Statistical Computing, Vienna, Austria (2009) ISBN 3-900051-07-0

20. Liaw, A., Wiener, M.: Classification and regression by randomforest. R. News 2(3), $18-22(2002)$ 